\title{
The Research of Anti-RGPO Algorithm of OW-MF Base on Detecting the Pulse Front Edge
}

\author{
Yang Guang, Lu Dawei, Yan Xingwei ,Zhang Jun \\ atr lab, school of electronic science and engineering \\ national university of defense technology \\ changsha, hunan , p.r.china, 410073
}

\begin{abstract}
Radar echo gating with slide windows that start from front edge of echo pulse with different length can make full use of target echo and depress the jamming. A novel Optimum Windowing- Matched Filter(OW-MF)anti-jamming algorithm is presented, which enables the capability to depress the RGPO of LFM seeker radar. The length of optimum window is determined by SJR and SNR required for a effective target detection. Simulations validate the algorithm and related conclusions.
\end{abstract}

Keywords-The pulse front edge detecting, Optimization window researching, Part matching filer, RGPO

\section{INTRODUCTION}

In modern electronic warfares, the radar systems are challenged by different kinds of jams, which come from not only the nature, such as broadcasting signal, thunder and static electricity, but also artificial jams. The LFM radar enables low probability of interception because of its large TBP (Time-Bandwidth Product), so the LFM seeker radar is hard to interfered. Because of its good capability to depress noise, low transmitting power, long range detection and low cost, the LFM radar is widely used. But the LFM radar system also suffers from some disadvantages, such as long coherent time, low velocity resolution, etc. The Towed Radar Active decoy (TRAD) have been widely equipped by modern aircrafts. For their excellent jamming performance the TRAD appeals successfully to the department of defense of many countries[1]. Realizing importance of the research of ways to anti-TRAD, lots of method of anti-TRAD are worked out, such as "memory gating method""using velocity instead of range slope method"'sent periodic pulse method"" pulse front edge tracking method" and so on [2]. This paper present a novel Optimum Windowing-Matched Filter (OW-MF) anti-jamming algorithm, which enables the capability to depress the Range Gate Pull Out (RGPO) to LFM seeker radar. The algorithm use a window to capture the signal of the target and depress the signal of the jam farthest, then to detect the target by incomplete matching filtering. The algorithm is validated by the numerical simulations.

The whole paper is arranged as follows. The basic signal model is presented in section II. And the novel algorithm to anti-jamming is detailedly dedicated in the following section. Then in section IV, the factor that affected the length of optimum window is analyzed. The simulation in the section
$\mathrm{V}$ validated the presented algorithm. The whole work is summarized in the last section.

\section{SIGNAL MODEL}

The LFM radar transmits chirp signal in every pulse, the pulse repetition interval is $T_{p}$, with $\tau$ is the pulse width . $t_{m}$ stands for slow time variable with the beginning time $t=m T_{p}$ for the $m^{t h}$ pulse, so the $m^{\text {th }}$ transmitted pulse can be expressed as below [3]

$$
e_{m}\left(t_{m}\right)=A \operatorname{Rect}\left(t_{m} / \tau\right) \cos \left(2 \pi f_{0} t_{m}+\pi K t_{m}^{2}+\varphi_{m}\right)(1)
$$

After the coherent mixing, amplifying and I/Q processing, the base band echo of the target in one pulse polluted with TRAD jams can be expressed as (2)

$$
\begin{aligned}
s_{m}\left(t_{m}\right) & =A_{T} \operatorname{Rect}\left(\frac{t_{m}-2 R_{T} / c}{\tau}\right) \cdot \exp \left\{j\left[K \pi\left(\left(1-\frac{2 v}{c}\right) t_{m}-\frac{2 R_{T}}{c}\right)^{2}-2 \pi f_{0}\left(\frac{2 v}{c} t_{m}+\frac{2 R_{T}}{c}\right)\right]\right\} \\
& \left.=A_{J} \operatorname{Rect}\left(\frac{t_{m}-2 R_{J} / c}{\tau}\right) \cdot \exp \left\{j\left[K \pi\left(1-\frac{2 v}{c}\right) t_{m}-\frac{2 R_{J}}{c}\right)^{2}-2 \pi f_{0}\left(\frac{2 v}{c} t_{m}+\frac{2 R_{J}}{c}\right)\right]\right\}
\end{aligned}
$$

where, $A_{T}$ is the target echo amplitude, $A_{J}$ is the jam echo amplitude which is relevant to the gain of jammer, $R_{T}$ is the slant range of target. And $R_{J}$ is the appearing range of jamming, $R_{J}$ is controlled by the time delay of jamming pulse which is decoyed by the transmitter of jammer. $R_{T}=R_{0}-v m T_{p}$ is the radar range of target of the $m^{\text {th }}$ period, $R_{0}$ is the initial range of the frame, $v$ is the velocity of the target relative to the radar in LOS. The range $R_{J}$ of TRAD with the constant velocity $v_{J}$ is (3)

$$
R_{J}=\left\{\begin{array}{cc}
R_{T} & t_{0} \leq t<t_{1} \\
R_{T}+v_{J}\left(t-t_{1}\right) & t_{1} \leq t<t_{2}
\end{array}\right.
$$

where, $t_{0} \leq t<t_{1}$ is TRAD stopping interval, $t_{1} \leq t<t_{2}$ is TRAD decoying interval, $t \geq t_{2}$ is TRAD closing interval. The relative delay between jam and target echo is $\Delta \tau=2\left(R_{J}-R_{T}\right) / c$. Because of the noise exiting in the receiver, the actual sampling signal is as (4)

$$
x_{m}\left(t_{m}\right)=s_{m}\left(t_{m}\right)+\varepsilon_{m}
$$

In this paper the only case that delay of jam shorter than that of pulse is considered, $\Delta \tau<\tau$. 


\section{THE ANTI-RGPO ALGORITHM OF OW-MF}

\section{BASE ON DETECTING PULSE FRONT EDGE}

\section{A. Pulse front edge detecting}

To filter or depress the jamming component of echo effectively is the basic problem of anti-jamming. As shown in the Figure 1, because the RGPO echo always arrives later than that of the target for the LFM radar[4], so a range window can be added to the echo at front edge of target echo to filter the power of the RGPO. At the beginning of RGPO echo, the target echo superposes the RGPO echo and then they separate from each other as time goes. In the range gate, the amplitude of target echo is polluted by the RGPO and can't be used to track target directly. But the separation of target and RGPO at the front edge provides jamming-free area which can be used to indicate the position of the target $\lceil 5\rceil$.

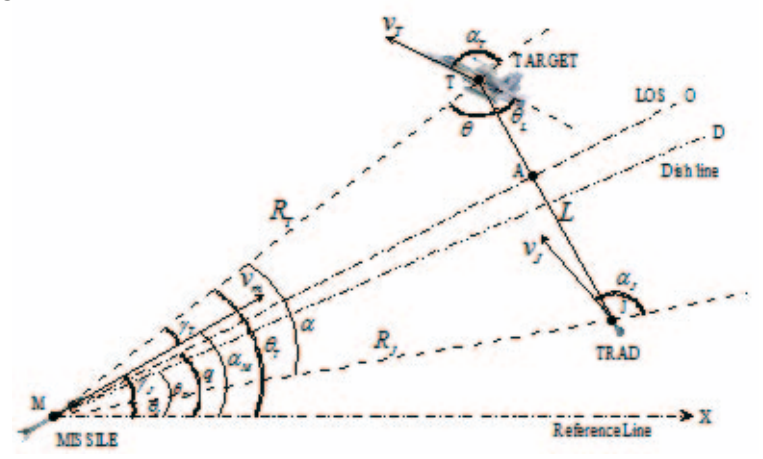

Figure 1. Sketch map for TRAD

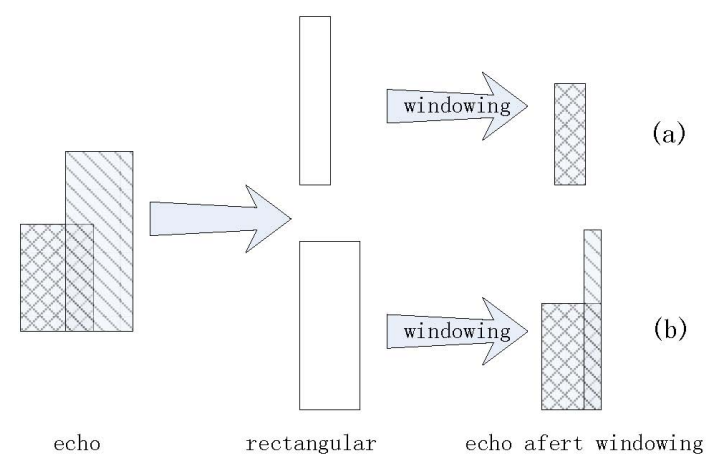

Figure 2. The sketch map of windowing for the echo

The front edge detecting process as (5)

$$
t_{m 0}=\min _{t} \operatorname{root}\left[\frac{d^{2}\left|\int_{0}^{t} x_{m}(\tau) d \tau\right|}{d t^{2}}\right]
$$

First of all, the received echo is integrated to get its envelop. By differentiating to the envelop signal, the stationary points can be achieved which include the target and jamming pulse front. The minimization of all stationary points is the required target pulse front which is the time of target echo arrival.

\section{B. Window the echo}

The window function $\mathbf{W i n}_{m}\left(t_{w}\right)$ is multiplied by echo at the front edge, $t_{w}$ is the length of the window, so the echo can be express as (6)

$y_{m}\left(t_{m}\right)=x_{m}\left(t_{m}\right) \cdot \mathbf{W i n}_{m}\left(t_{w}\right)$

There will be three cases with repected to the different length of window, as (7) shown

$$
\left\{\begin{array}{c}
t_{w} \leq \Delta \tau \\
\Delta \tau \leq t_{w}<\tau \\
t_{w} \geq \tau
\end{array}\right.
$$

When $t_{w} \geq \tau$, target echo amplitude has nothing to do with the length of window, and only more jam power be imported. The anti-RGPO algorithm fails in this situation. So the situation of $t_{w}<\Delta \tau$ will not be considered, other two cases will be discussed detailedly.

In the situation $t_{w} \leq \Delta \tau$, as shown in the Figure 2(a), the target echo will be restained without jam signal after windowing, so the echo in this time can be expressed as (8)

$$
y_{m}\left(t_{m}\right)=A_{T} \operatorname{Rect}\left(\frac{t_{m}-2 R_{T} / c}{t_{w}}\right) \cdot \exp \left\{j\left[K \pi\left(\left(1-\frac{2 v}{c}\right) t_{m}-\frac{2 R_{T}}{c}\right)-2 \pi f_{0}\left(\frac{2 v}{c} t_{m}+\frac{2 R_{T}}{c}\right)\right]\right\}+\varepsilon_{m}
$$

According to the theory of matching filtering, the unit impulse response of the matching filter as the expression (9)

$$
h_{m}\left(t_{m}\right)=\operatorname{Rect}\left(\frac{t_{m}}{\tau}\right) \exp \left\{-j \pi K t_{m}^{2}\right\}
$$

The output of the matching filter as (10)

$$
g_{m}\left(t_{m}\right)=y_{m}\left(t_{m}\right) \otimes h_{m}\left(t_{m}\right)
$$

The echo contains only target signal and noise without jamming echo after windowing. Changing the length of the window until the output satisfy the requirement for detecting, the window with this length will be the optimum. The length of the window should not be too short, because it will deprave matching filtering which will cause heavily mismatch, leading to the output never satisfy the requirement for detecting. So there is a minimum of $t_{w}$ that make the output meet the requirement of detection while not involving heavy mismatching problem.

In the situation of $\Delta \tau \leq t_{w}<\tau$, as shown in Figure 2(b), $t_{w}$ is larger than the RGPO echo delay time $\Delta \tau$, part of jam signal will be included within the windowing signal, so the echo can be expressed as (11)

$$
\begin{aligned}
y_{m}\left(t_{m}\right) & \left.=A_{T} \operatorname{Rect}\left(\frac{t_{m}-2 R_{J} / c}{t_{w}}\right) \cdot \exp \left\{j\left[K \pi\left(1-\frac{2 v}{c}\right) t_{m}-\frac{2 R_{T}}{c}\right)^{2}-2 \pi f_{0}\left(\frac{2 v}{c} t_{m}+\frac{2 R_{T}}{c}\right)\right]\right\} \\
& \left.+A_{J} \operatorname{Rect}\left(\frac{t_{m}-2 R_{T} / c}{t_{w}-\Delta \tau}\right) \cdot \exp \left\{j\left[K \pi\left(1-\frac{2 v}{c}\right) t_{m}-\frac{2 R_{J}}{c}\right)^{2}-2 \pi f_{0}\left(\frac{2 v}{c} t_{m}+\frac{2 R_{J}}{c}\right)\right]\right\}+\varepsilon_{m}
\end{aligned}
$$

Let the windowed echo pass the matching filter in (9) as above. For the echo contains both target signal and jam signal, the longer window takes, the more jam signal power is imported. And jam power will exceed target signal power as the window is becoming longer, which doesn't achieve 
the purpose to depress the jam. So the length of the window should not be too long, i.e.there is a maximum window length.

Summarizing above all, there are minimum and maximum lengths for the range window, the window length within $\left[t_{w \min }, t_{w \max }\right]$ is called the optimum window interval, which will ensure that the SNR or SJR satisfy the detection requirement.

\section{Incomplete matching filter}

For convenience, in this subsection the progress of incomplete matching filtering will be discussed [6]. $\eta(0<\eta<1)$ is ratio of that the width of pulse within the window divided by the width of pulse, then incomplete matching filtering is discussed with the influence of $\eta$. Suppose the sample echo after windowing is (12)

$$
s_{r}\left(t-t_{0}\right)=\operatorname{Rect}\left(\frac{t-t_{0}}{\eta \tau}\right) \exp \left\{j \pi K\left(t-t_{0}\right)^{2}\right\}
$$

Where $t_{0}$ is the arriving time of target. The matching filter will be

$$
h(t)=\operatorname{Rect}\left(\frac{t}{\tau}\right) \cdot \exp \left\{-j \pi K t^{2}\right\}
$$

The output can be simplified as (14) when $T B P \geq 100$ which is reasonable in most applications.

$$
\left|s_{\text {out }}(t)\right|=s_{r}(t) \otimes h(t) \approx \eta \tau \operatorname{Rect}\left(\frac{t}{(1+\eta) \tau}\right) \operatorname{sinc}(K \eta \tau t)
$$

The output for the situation without windowing can be expressed as (15)

$$
s_{\text {out }}^{\prime} \approx \tau \operatorname{sinc}(K \tau t)
$$

Comparing (14) and (15), owing to the influence of windowing, the amplitude of matched filter output descends $\eta$ times than that of original, and the width of main lobe

become $1 / \eta$ wider than that of original, but the target position holds after windowing.

\section{SIMULATION}

For the scenario of RGPO, the target range is $3000 \mathrm{~m}$, the length of the TRAD line is $150 \mathrm{~m}$ (the greatest range between target and the RGPO equipment is $150 \mathrm{~m}$ ). The carrier frequency is $17 \mathrm{GHz}$ for the LFM radar, the bandwidth is $16 \mathrm{M}$, pulse width is $2 \mu \mathrm{s}$, pulse interval is $T_{p}=40 \mu s$, sampling frequency is $F_{s}=80 \mathrm{MHz}$, sampling interval is $T_{s}=1 / F_{s}$. The simulation is done respectively for the jam echo delay of $10 T_{s} \sim 160 T_{s}$ and the SJR of $-20 d B \sim 0 d B$, the detecting threshold is $-13 d B$ for SNR, the rectangular window, hamming window and gauss window are used.

The result of the simulation is shown as the Figure $3 \sim$ Figure 5, Figure 3 shows the SNR/SJR varifying corresponding to different window length after MF, we can see the performance of gauss window and hamming window is better than that of rectangle window. Figure 4 shows the interval of the optimum window length changing with different jam delay. As shown in the figure, large delay leads to larger range of the optimum window length, and the gauss window and hamming window have larger range of optimum window length than that of rectangle one. Figure 5 shows the connection of the range of optimum window length and the SJR. The larger SJR is, the wider the range of the optimum window length will be. And the range of the gauss window and hamming window is larger than that of rectangle. As signal after rectangle windowing preserves more jamming power than that of hamming and gussian window, so the gauss window and hamming window have a larger range of window length with the same input SJR than that of rectangle.

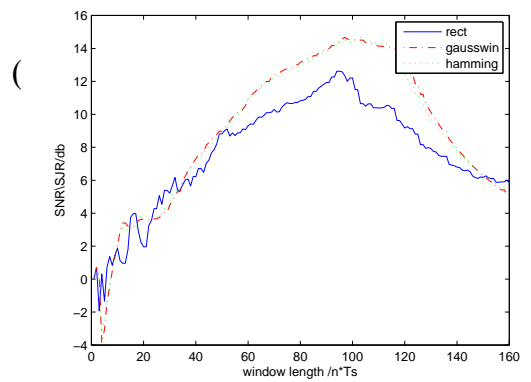

Figure 3. the connection of SNR/SJR and window length after filtering

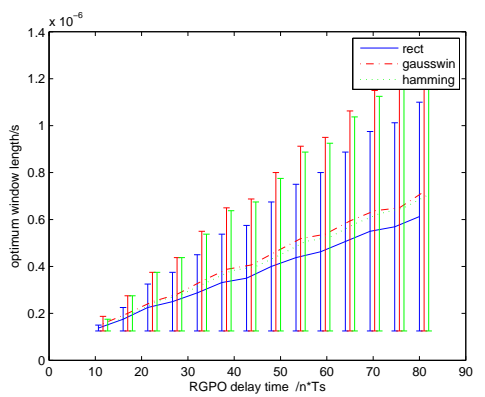

Figure 4. chart of the connection of the range of optimum window length and the RGPO echo delay

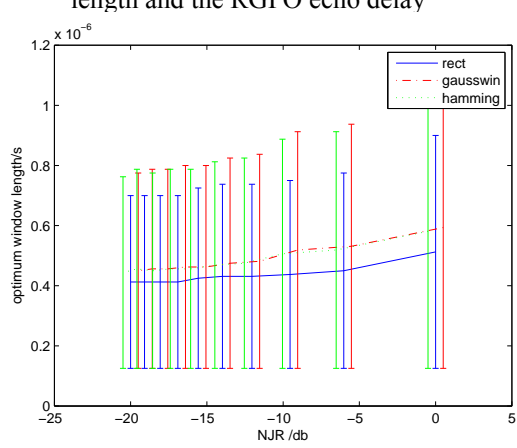

Figure 5. chart of the connection of the rangeof optimum window length and the input SJR 


\section{SUMMARIES}

This paper presents an anti-RGPO algorithm base on the front edge detection OW-MF. This method uses the windowing technique to depress the power of RGPO, and make sure the peak of RGPO lower than the threshold to achieve the requirement of detection. The simulation shows the feasibility of presented algorithm. Choosing the appropriate window length can depress the jam or even clear the jam. Factors that affect the performance of the algorithm are analysed, such as the delay echo of the jam initialization of the SJR and the different shape of the window. The range of the optimum window length supposes the direct proportion, connected with the jam echo delay and the initialization of the SJR. Range of the optimum window length using the gauss and hamming window is wider than that of rectangle one. And the simulations validate our antiRGPO algorithm.

\section{ACKNOWLEDGEMENTS}

This project was supported by the Advanced Research Foundation of National Defence

\section{REFERENCES}

[1] GAO Bin, MAO Shi-yi, SUN Jin-ping. "Effect of towed-decoys against antiaircraft missile with monopulse seeker[J]".Systems Engineering and Electronics, 2010, 32(11), pp2394-2397.

[2] QU Chang-wen. "The Method of Anti-jam Technology for Decy[J]".Spaceflight electron antagonize, 1995(3).

[3] HE Song-hua,HUANG Da-hua,ZHANG Jun. "A Study on Detection of Range-eclipsed Target in LFM Pulse Compression Radar[J]". Modern Radar,2009, 31(3), pp14-17.

[4] BAI Wei-xiong, JIAO Guang-long, FU Hong-wei. "Study on antagonistic technology of towed decoys[J]". Systems Engineering and Electronics, 2009,31(3), pp579-582.

[5] ZHANG Xiao-lin, SHEN Fu-min, LIU Zheng. "A Valid Method for Terminal Guidance Radar to Counter Range Gate Pull Off'. Guidsnce and Fuze,2003, 24(4), pp46-49.

[6] Ian G. Cumming, Frank H. Wong. "Digital processing of synthetic aperture radar data:Algorithms and Implementation[M]".publishing house of electronics industry, 2007, pp71-72. 